\title{
Using genetic algorithms for real-time object detection
}

\author{
J. Martínez-Gómez ${ }^{1}$, J.A. Gámez ${ }^{1}$, I. García-Varea ${ }^{1}$, V. Matellán ${ }^{2}$ \\ Computing Systems Department ${ }^{1}$, Dept. of Mechanical and Computer Engineering ${ }^{2}$ \\ University of Castilla-La Mancha ${ }^{1}$, University of Leon ${ }^{2_{2}}$ \\ \{jesus_martinez, jgamez, ivarea\}@dsi.uclm.es ${ }^{1}$, vicente.matellan@unileon.es ${ }^{2}$
}

\begin{abstract}
This article presents a new approach to mobile robot vision systems based on genetic algorithms. The major contribution of the proposal is the real-time adaptation of genetic algorithms, which are generally used offline. In order to achieve this goal, the execution time must be as short as possible. The scope of the System is the robotic football competition Robocup ${ }^{4}$, whitin the Standard Platform category ${ }_{\bar{\alpha}}$ The system developed detects and estimates distance and orientation to key elements on a football field, such as the ball and goals. Different experiments have been carried out whitin an official Robocup environment.
\end{abstract}

\section{Introduction}

For mobile robotics, image processing is, one of the most important elements. Intelligent robots need to retrieve information from the environment in order to interact with it. Vision cameras are one of a robot's key devices. The images taken by the robot's camera need to be processed in real time with limit processing resources. The systems developed need to cope with noisy and low quality images, and in order to process the maximum number of images by second, the algorithms must be as efficient as possible.

Different_solutions have been proposed over the last years. These proposals use the information obtained with colour filtering processes [1]. One of the most widely-used solutions for the Standard Platform category in the Robocup[2] are scan-lines [3]-_The approach presented here carries out object recognition by using real-time genetic algorithms[4].

The number of iterations and individuals for the genetic algorithm must be reduced as much as possible in order to improve efficiency. This is necessary because the system has to be applied in real time. In order to prevent system performance from being affected by this reduction, the individuals will be initialized using all the available information. This initial information can be obtained from previous populations and $a_{\lambda}$ colour filtering process, applied to the last image taken by the robot's camera. After an image showing an object $o$, the next image has a high probability of showing the same object. The information obtained from previous populations allows us to take advantage of the high similarity between consecutives images taken by the camera.

\footnotetext{
${ }^{1}$ http://www.robocup.org/
} 
Some authors propose the use of cellular genetic algorithms[5] instead of reducing the number of individuals and iterations. These algorithms obtain a good performance for optimization tasks that need high efficiency with a reduced number of iterations.

Our hypothesis i $\overline{\bar{v}}_{\mathrm{t}}$ the similarity between captured images, and the information obtained with the filtering process, can be used to develop a real-time vision system based on genetic algorithms. Different tests in real scenarios using the biped robot Nao[6] have been carried out to evaluate our proposal. These tests show the object (ball and goals) recognition process on the official Robocup football field.

The article is organized as follows: Section 2 is dedicated to genetic algorithms, and the image processing process is outlined in Section 3. We describe the full vision system in Section 4, and in Section 5 we explain the experiments performed and the results obtained. Finally, the conclusions and areas for future work are given in Section 6 .

\section{Genetic Algorithms}

Genetic algorithms (GAs) are search techniques included in bio-inspired algorithms. These algorithms carry out steps inspired by evolutionary biology. A population of individuals is kept throughout the process. The individuals represent potential solutions for the problem we want to solve. The process involves certain iterative steps: selection of the individuals to reproduce, crossover, mutation, replacement and evaluation of the population. The algorithm terminates when a maximum number of iterations has been executed or when a satisfactory fitness value has been reached.

GAs are usually applied to solve optimization problems with a large search space. The computational cost of these algorithms is considerable due to the high number of iterations and individuals. The individuals of the population scan the search space. In the course of the iterations, best individuals propagate their information to the new generations. The algorithm converges while the population is evolving. The evaluation of individuals is one of the key points of GAs. This evaluation obtains a fitness value for each individual. This numeric value will be greater for the best individuals, according to the solution that is being searched for.

\section{Problem restrictions}

The vision system has to be valid for use in the Standard Platform category. Robot Nao-is the official platform for this category, and its camera takes 30 (320 x 240 pixels) frames per second. The camera's native colour space is YUV[7]. In order to reduce the amount of information to work with, the captured images are filtered. This processing removes the pixels that do not pass a colour filter. The key colours in the Robocup environment are yellow and blue for the goals, green for the carpet, orange for the ball and white for the field lines. Football player equipment is red and dark blue. The filtering is carried out by defining a top and bottom limit for the $\mathrm{Y}, \mathrm{U}$ and $\mathrm{V}$ colour components. A pixel will successfully pass a filter only if all its components are between these limits. Fig. 1 shows a filtering example for blue. 


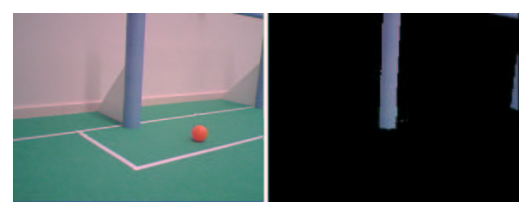

Fig. 1. Colour filtering for the blue goal

Object recognition has to be carried out during a football match. The environment includes objects that are partially hidden behind others, so the frames taken in a football match will not always show the complete object we want to recognize. Scan-line-based methods present a lot of problems in these situations, whereas our system works properly, as will be shown in the results section.

\section{Vision System}

Genetic algorithms keep-individuals that represent potential solutions to problems. For our vision system, individuals have to represent the detection of the object $o$ placed at distance $d$ with the orientation or. This information (object $o$ at distance $d$ with orientation or ) is contrasted with the entrom the last frame captured by the robot's camera. The fitness will be high for individuals with information that is plausible with respect to the last image. On the other hand, the fitness will be low if the object $o$ does not appear in the image.

\subsection{General processing scheme}

The processing begins, with the arrival of new images at the robot's camera. A new image will evolve a new population for each object to be recognized. Three diste are blue goal, the yellow goal and the orange ball), so three different populations will be kept. After taking a new image, the colour filtering allows the robot to know the objects likely to appear in the image. The populations of the non-plausible objects will not be evolved. Fig.2 shows the complete general processing scheme.

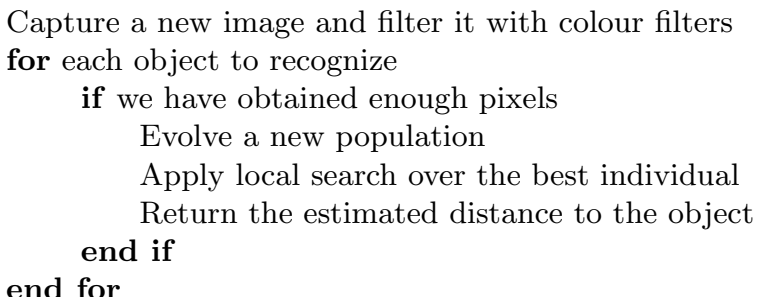

Fig. 2. General system processing scheme

In order to avoid local optimums, the population will be restarted after a given number of iterations failing to improve the best individual. An iteration will increase the value of a counter if the best fitness of the iteration is not greater than the best global fitness. The counter value will be set to zero if the iteration obtains the best fitness. The population will be restarted if the counter reaches a limit value. 


\subsection{Genetic Representation}

In addition to the distance between the camera and the object to be recognized, we also need to estimate the orientation between both elements. This information is not only needed for self-location tasks [8], but also for the application of the fitness function using the images taken with the robot's camera. The shape of an object in an image will depend on the distance and the orientation between object and camera.
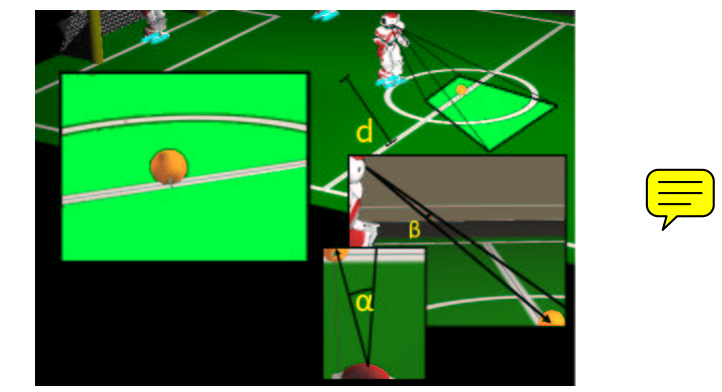

Fig. 3. Image taken with specific distance and orientation between object and camera

Fig. 3 shows an image taken when the distance between camera and object is $d$ and the difference of orientation is $\alpha$ in the $x$-axis and $\beta$ in the $y$-axis. With the same distance $d$ and different $\alpha$ or $\beta$ values, captured images will show the same ball but located at a different position within the image. The image will not show the ball with big $\alpha$ or $\beta$ variations. A third component for the

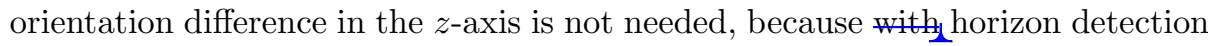
techniques[9], the image can be processed to show all the objects parallel to the floor. Each individual stores the following information (genes):

- Distance to the robot: $d$

- Orientation difference in the $x$-axis: $\alpha$

- Orientation difference in the $y$-axis: $\beta$

All the genes are represented by a numerical value, limited by the maximum distance detection for $d_{\text {-and }}$ by the field of view for $\alpha$ and $\beta$. An additional gene is needed to perform goal detection. This gene $(\theta)$ represents the goal orientation when the frame is taken. Two frames taken with the same $<d, \alpha, \beta>$ parameters will be different if the goal orientation varies, as can be observed in Fig.4.
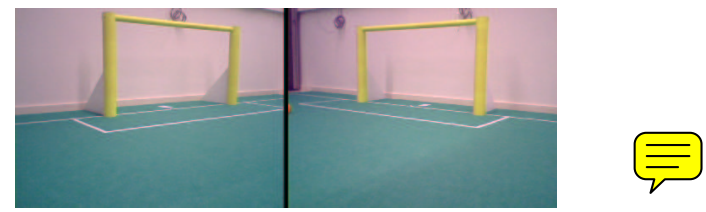

Fig. 4. Images taken varying the $\theta$ parameter

\subsection{Obtaining the $\beta$ parameter}

We can avoid modelling $\beta$ if we know the angle between the camera and the floor in the $y$-axis, $\gamma$. Thus $\beta$ can be calculated using $\gamma$, the distance $d$, and the 
orientation in the $x$-axis $\alpha$. With this approach, the areas of the search space that represent unreal solutions will not be explored. With $\gamma$ and the camera's field of view, we can obtain the minimum and maximum distances at which we can detect elements. If $\gamma$ is close to 90 degrees, the robot will be able to recognize distant objects, but not a nearby ball.

The main problem of calculating $\beta$ instead of modelling it is that our algorithm will heavily depend on $\gamma$ estimation. The performance of the algorithm will decrease if $\gamma$ is not correctly estimated. For legged robots, the movement of the robot causes an enormous variation in the camera angle, which makes it difficult to obtain a precise value for $\gamma$. For wheeled robots, the movement will not affect the camera angle as much as for legged ones and $\gamma$ can be accurately calculated.

\subsection{Fitness function}

The fitness function returns numeric values, according to the goodness, of the projection obtained with the parameters $\langle d, \alpha, \beta\rangle$ of each individual. To evaluate an individual, its genes are translated into a projection of the object that the individual represents. The projection needs a start position $\langle x, y\rangle$, obtained from $\alpha$ and $\beta$. The size of the object depends on $d$.

An object projection is evaluated by performing a comparison between it and the information obtained from the filtering process. A pixel $\langle x, y\rangle$ of the projection will be valid only if the pixel $\langle x, y\rangle$ of the image captured by the robot's camera successfully passes the colour filter. This evaluation is shown in Fig. 5, where the left image shows the original image after an orange filter. The right one shows the result of evaluating 12 different individuals, where red pixels are invalid (they have not passed the colour filter) and green pixels are valid.

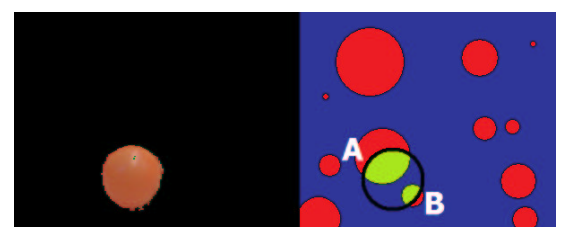

Fig. 5. Filtered image (left) and evaluation of 12 individuals to detect the ball (right)

With the processing we obtain the number of valid and invalid pixels for each individual. Using the percentage of pixels that pass the filter as a fitness function has a serious drawback: individuals representing distant objects obtain

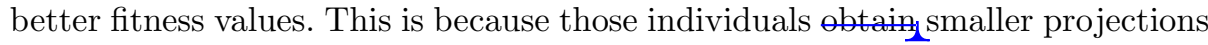
with a $_{\mathbf{\Omega}}$ higher probability of having a bigger percentage of valid pixels $\bar{\beth}_{\bar{\imath}}$ Due to this problem, and using the information obtained with the filtering, the seled fitness function veturns the minimum value of:

- $\%$ of pixels of the projection that have passed the colour filter.

- $\%$ of pixels that passed the colour filter and belong to the valid projection pixels.

We_will study individuals A and B in Fig. 5 to illustrate the behaviour of the function. Individual $\mathrm{B}$ has a higher percentage of pixels that passed the filter (70 versus 45 ). On the other hand, only $5 \%$ of the pixels that passed the orange filter belong to individual B. For A, this percentage rises to $35 \%$.

$\leftarrow \begin{aligned} & \text { aquí habría que } \\ & \text { completar }\end{aligned}$




\subsection{Population initialization}

For genetic algorithms, the population is usually randomiy initialized. Our approach uses additional information to initialize the first individuals. Firstly, we $\mathrm{ean}_{\Omega}$ use individuals from populations of previous captures. In addition to this, the information extracted from the filtering process can-be used. Such information is the number of pixels of each colour, and the $x$ and $y$ component of the centroid of the distribution of pixels obtained with the colour filter. With this information, an individual can be initialized in three different ways:

- Randomly.

- Using the information from the filtering process.

- Cloning an individual from a previous population.

The first two ways of generating a new individual can always be used. The third one can only be used when a valid population is available. Such population must have been evolved to recognize the same object $o$ that we want to recognize. The number of frames between the current one and the last one that evolved a population to recognize $o$ has to be small. If the frame number difference is large, the individuals of the population will not be valid for cloning. A draw is carried out to select the way in which an individual is initialized. All the ways have a probability that depends on the number of frames from the last frame that recognized the object we are studying. We need two parameters to obtain these probabilities:

- $M W$ : Max probability of cloning an individual from a previous population.

- MNF: Max number of frames possible between the present frame and the last one that recognized the object we are studying.

The sum of the three probabilities_must be 1.0. The probability of initializing individuals by cloning them from other populations will decrease if the number of frames without updating $(N F W U)$ that population increases.

$$
\text { CloneProb : } M W-M W *(N F W U / M N F)
$$

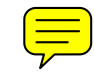

Le ha pasado algo a esas líneas.
Shis value must be between 0 and 1 . The rest of the probability will be distributed between the initialization using initial information or randomly:

$$
\begin{array}{r}
\text { InitialInfoProb }:(1-(\text { CloneProb })) * 0.66 \\
\text { RandomlyProb }:(1-(\text { CloneProb })) * 0.33
\end{array}
$$

If we increase the number of individuals that are randomly init variety of the initial population will be greater. Using the initial i decir que lo pones the algorithm's elitism will increase (with the problem of local optim a mano porque 1/3 individuals cloned from other populations, the algorithm will con $2 / 3$ with small variations between frames. The balance between elitism an can be obtained through a correct combination of these three ways.

\subsection{Partial object occlusion}

Vision systems must cope with hard environments. For example, the objects to recognize can be partially hidden behind other robots, or the images captured 
by the robot's camera may show only parts of the desired object, due to the camera's orientation. Our proposal performs the individual's evaluation using the entire object's projection and not partial characteristics. This is the reason that our system works properly with occlusions.

In the presence of obstacles between the camera and the o evaluation function will assign lower fitness values to the the algorithm will recognize the object. Fig.6 shows an in partially captured.

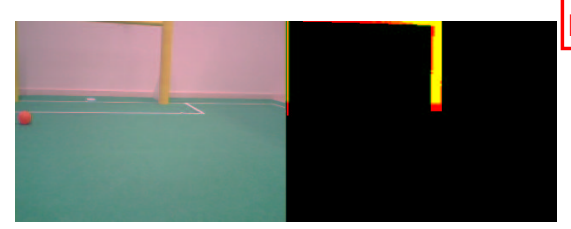

esto tienes que

repetirlo en las conclusiones. Es la parte buena (date más autobombo :-)

Fig. 6. Object partially captured

\section{Experiments and results}

The experiments were carried out on a Robocup Standard Platform football field, with the official goals, a $6 \times 4$ metre carpet and a ball. We used a Nao robot, taking 2 images per second. The format of the images is YUV and their size is $320 \times 240$ pixels. While the experiments were being carried out, the absolute difference between the real and estimated distance to the object we wanted to detect was stored per frame. The estimated distance was the value of the $d$ gene of the individual with the best fitness. Lighting conditions were stable throughout the experiments, and the colour filters were optimal.

The execution time for each image was variable. We decided to use two frames per second because the maximum execution time was never greater than 450 milliseconds. The execution time to perform a single detection can be split into different parts. First the image taken by the camera is filtered, then the necessary populations are evolved, and finally local search is applied to the best individuals.

\subsection{Genetic algorithm parameters}

The experiments were carried out with the following parameters:

- Individual number: 12 and Iteration number: 24

- Mutation probability: $5 \%$ and Crossover type: point

- Replacement: generational algorithm

- Restart after 25\% iterations without improving the global optimum

- MW: 0.5 and $M N F: 10$

The algorithm uses a limited number of individuals and iterations. The mutation probability and the crossover type are standard, and the entire population is replaced with the offspring at the end of the iteration. Due to this the quality of the population can decrease while the iterations are executed.

After evolving the population, a simple local search process (Hill Climbing) is applied to the best individual. This processing will allow us to improve the best fitness. The local search is applied by evaluating positive and negative variations for the genes of the individual. The algorithms that combine concepts and strategies from different metaheuristics are called memetic algorithms [10]. 


\subsection{Experiment 1 - Hypothesis validation}

The objective of the first experiment was to prove that the system is able to work in real time, recognizing the environment elements and estimating the distance to them. We used the standard parameters described above and we executed the same tour over the football field 6 times. 30 frames were taken per tour ( 15 seconds). The frames captured the yellow goal placed between 360 and $300 \mathrm{~cm}$, and the orange ball placed at distances between 260 and $200 \mathrm{~cm}$.

The experiment consisted of 180 different frames $(6 \times 30)$. We stored the absolute difference between real and estimated distance (denoted $D B R E D$ ) and the fitness of the best individual of the population by frame. These fitness values were used to generate different data sets. Each one of these data sets had only the detections carried out with individuals whose fitness values were greater than certain thresholds. Table 1 shows, taking the ball and yellow goal separately, and with four different threshold values $(0,0.25,0.5$ and 0.75$)$, the average of the $D B R E D$. It also gives the percentage of frames that obtained an individual with a fitness value greater than the threshold.

Table 1. Average $D B R E D$ and $\%$ of frames with a fitness value over certain thresholds

\begin{tabular}{|l|r|cccc|ccccc|}
\hline Fitness & \multicolumn{3}{|c|}{$>0.0$} & $>0.25$ & $>0.5$ & $>0.75$ & \multicolumn{5}{|c|}{$>0.0$} & $>0.25$ & $>0.5$ & $>0.75$ \\
\hline Average $(\mathrm{cm})$ & Ball & 42.62 & 40.57 & 31.77 & 22.75 & Yellow & 40.03 & 37.88 & 33.1 & 32.69 \\
Frames (\%) & & 68.89 & 68.33 & 57.78 & 8.89 & Goal & 99.44 & 93.33 & 44.44 & 8.89 \\
\hline
\end{tabular}

It can be seen that the fitness function properly represents the goodness of the individuals. This is because using individuals with higher fitness values reduced the average of the differences between real and estimated distances. Table 2 shows the percentage of frames that obtained a difference between estimated and real distance lower than certain thresholds.

Table 2. Percentage of frames that obtained a $D B R E D$ lower than certain thresholds

\begin{tabular}{|c|c|c|c|}
\hline & $\begin{array}{l}\text { Percentage of frames under } \\
100 \mathrm{~cm} 75 \mathrm{~cm} 50 \mathrm{~cm} 30 \mathrm{~cm}\end{array}$ & & $\begin{array}{l}\text { Percentage of frames under } \\
100 \mathrm{~cm} 75 \mathrm{~cm} 50 \mathrm{~cm} 30 \mathrm{~cm}\end{array}$ \\
\hline Ball & $\begin{array}{llll}63.63 & 56.11 & 44.44 & 35.55\end{array}$ & Yellow Goal & $\begin{array}{lll}87.78 & 72.22 & 51.67\end{array}$ \\
\hline
\end{tabular}

The results obtained show a high degree of robustness, especially for the yellow goal. In an environment with a maximum distance of $721 \mathrm{~cm}$, a high (37.37\% and $51.67 \%)$ percentage of frames obtained differences for the distance estimation under 30 centimetres.

Ball recognition (with our genetic algorithm) was more complicated than goal recognition. This is why only individuals which are very close to the global maximum (perfect detection) obtain fitness values different from zero. Due to the small size of the ball in the frames captured, only the projections of individuals close to the final solution have pixels in common with the image obtained after the colour filtering process. The convergence of a genetic algorithm with these characteristics will not be constant. $83.83 \%$ of correct ball recognitions (fitness $>0$ ) were carried out with fitness values greater than 0.5 . For the goal, this percentage descends to $44.69 \%$. 


\section{$5.3 \quad$ Experiment 2 - $\beta$ study}

The main objective of the second experiment was to test whether the $\beta$ parameter can be calculated using the other parameters. The performance of the algorithm

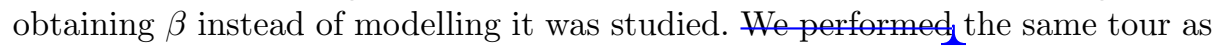
in experiment 1 .

For this experiment, the individuals did not use the $\beta$ gene, but the parameter is needed for the fitness function and has to be calculated. This was done using the parameters $d$ (distance to the object), $\alpha$ (orientation difference in the $x$-axis) and $\gamma$ (orientation difference between the robot's camera and the floor in the $y$-axis). $\gamma$ is obtained using the robot's sensors. The experiment consisted of 180 frames again and results obtained are shown in table 3 .

Table 3. Average $D B R E D$ and $\%$ of frames with a fitness value over certain thresholds

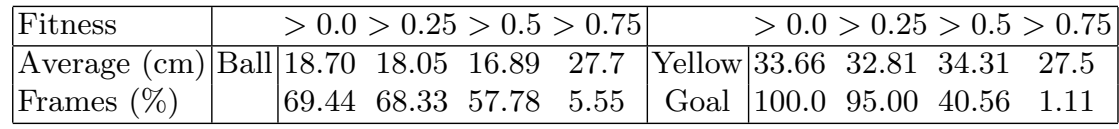

The first conclusion drawn from the results is that the number of correct detections (frames that obtained fitness values greater than 0 ) increased $_{2}$ However, the percentage of frames with a fitness value greater than 0.5 and 0.75 decreased. This is because modelling $\beta$ instead of obtaining it from the other parameters the algorithm to reach situations that are not according to the model, but which are valid due to noise or the difference between the real and estimated $\gamma$ value.

The average difference between the real and estimated distance $(D B R E D)$ decreased considerably. With fewer number of genes and the same iterations, $\overline{\overline{\bar{\tau}}}{ }_{\mathrm{S}}$ converge faster to better solutions. In order to establish a complete comparison between modelling $\beta$ and calculating it with other parameters, table 4 provides the percentage of frames that obtained a $D B R E D$ lower than certain thresholds.

Table 4. Percentage of frames that obtained a $D B R E D$ lower than certain thresholds

\begin{tabular}{|c|c|c|c|}
\hline & $\begin{array}{l}\text { Percentage of frames under } \\
100 \mathrm{~cm} 75 \mathrm{~cm} 50 \mathrm{~cm} \quad 30 \mathrm{~cm}\end{array}$ & & $\begin{array}{l}\text { Percentage of frames under } \\
100 \mathrm{~cm} 75 \mathrm{~cm} 50 \mathrm{~cm} \quad 30 \mathrm{~cm}\end{array}$ \\
\hline Ball & $\begin{array}{llll}68.89 & 68.89 & 65.56 & 54.44\end{array}$ & Yellow Goal & 96.6793 .3376 .67 \\
\hline
\end{tabular}

If we compare table 4 and 2 , we can see that the robustness of the algorithm has improved. The faster convergence of the algorithm with fewer number of genes makes it possible to obtain a higher percentage of frames with a small $D B R E D$ to the object.

The main conclusion drawn from the data is that the gene number should always be as small as possible. If one of the parameters that are modelled can be obtained from other parameters, this parameter should be removed. In order to use fewer genes, we have to use all the possible information retrieved from the environment, the platform and the elements to recognize. This information allows us to include our knowledge about the problem in the algorithm, and with such information the algorithm will only reach individuals representing real situations (according to the robot and the environment). 


\subsection{Experiment 3 - $M W$ study}

The third experiment shows how $M W$ affects the vision system. This parameter defines the maximun probability of cloning an individual for initialization from previous populations. $M W$ defines the weight of previous frames for the process. If the value of this parameter increases a higher number of individuals from the initial population will represent solutions reached for previous frames.

The robot captured 20 different images from a static position. While the frames were being captured, the robot's camera orientation was quickly varied. All the frames show the blue goal placed at $250 \mathrm{~cm}$ and the orange ball situated at 150 $\mathrm{cm}$. Due to the camera movements (only the orientation changed), most of the frames only partially show these elements. We used the standard parameters for the genetic algorithm, and s was $m$ Todas con letra 0 elled as a gene. The variations in $M W$ defined the different configurations. Con número experiment was repeated 9 times witheach different configurations to obtain a final set of 180 frames $(20 * 9)$. Four different configurations were tested, with $M W$ values of $0,25,50$ and $75 \%$. Table 5 shows the results obtained for the experiment.

Table 5. Average DBRED and \% of frames with a fitness value over certain thresholds

\begin{tabular}{|c|c|c|c|c|c|c|c|c|c|}
\hline & $M W$ & Fit $>0$ & Fit $>0.2$ & Fit $>0$. & Fit $>0.75$ & Fit $>0$ & it $>0.2$ & Fit $>0.5$ & it $>0.75$ \\
\hline \multirow{4}{*}{ Ball } & 0.00 & 47.37 & 47.37 & 36.93 & 31.75 & 93.59 & 93.59 & 78.84 & 35.26 \\
\hline & 0.25 & 43.10 & 41.43 & 34.26 & 34.27 & 91.66 & 91.02 & 80.12 & 44.87 \\
\hline & 0.50 & 41.37 & 41.26 & 33.63 & 33.67 & 89.74 & 89.10 & 75.64 & 29.49 \\
\hline & 0.75 & 43.48 & 42.08 & 32.72 & 33.49 & 89.10 & 87.18 & 75.00 & 32.69 \\
\hline \multirow{4}{*}{$\begin{array}{l}\text { Blue } \\
\text { Goal }\end{array}$} & 0.00 & 58.02 & 49.48 & 27.15 & 12.78 & 100.0 & 82.68 & 47.49 & 12.85 \\
\hline & 0.25 & 53.64 & 42.63 & 26.71 & 19.72 & 98.32 & 83.80 & 56.42 & 13.97 \\
\hline & 0.50 & 51.22 & 43.54 & 21.76 & 14.16 & 98.88 & 87.71 & 55.87 & 13.97 \\
\hline & 0.75 & 44.16 & 37.60 & 24.45 & 15.39 & 98.88 & 89.94 & 64.25 & 12.85 \\
\hline
\end{tabular}

We can observe how the changes applied to $M W$ do not produce big variations in the difference between the real and estimated distance. Table 5 shows how the percentage of frames that obtained better fitness values increases with greater $M W$ values. For the blue goal, this happens for all the $M W$ values. For the ball, the optimum point for the $M W$ value is 0.25 . The performance of the algorithm gets worse if $M W$ is greater than 0.25 .

Table 6. Percentage of frames that obtained a DBRED below certain thresholds

\begin{tabular}{|c|c|c|c|c|c|c|c|}
\hline & \multirow[b]{2}{*}{$M W$} & \multicolumn{2}{|c|}{ Percentage of frames under } & & \multirow[b]{2}{*}{$M W$} & \multicolumn{2}{|c|}{ Percentage of frames under } \\
\hline & & $100 \mathrm{~cm} 75 \mathrm{~cm} 50 \mathrm{~cm}$ & $30 \mathrm{~cm}$ & & & $100 \mathrm{~cm} 75 \mathrm{~cm} 50 \mathrm{~cm}$ & $30 \mathrm{~cm}$ \\
\hline \multirow{4}{*}{ Ball } & 0.00 & 82.6972 .4462 .18 & 33.33 & \multirow{4}{*}{$\begin{array}{l}\text { Blue } \\
\text { Goal }\end{array}$} & 0.00 & $\begin{array}{lll}77.09 & 68.7155 .31\end{array}$ & 32.96 \\
\hline & 0.25 & $85.90 \quad 79.4971 .79$ & 31.41 & & 0.25 & $81.00 \quad 73.7462 .57$ & 34.08 \\
\hline & 0.50 & $85.90 \quad 75.0067 .31$ & 35.30 & & 0.50 & 81.5675 .4161 .45 & 43.01 \\
\hline & 0.75 & $80.77 \quad 73.72 \quad 64.10$ & 32.69 & & 0.75 & $87.15 \quad 78.77 \quad 72.07$ & 48.60 \\
\hline
\end{tabular}

Finally, table 6 presents the percentage of frames that obtained differences between the real and estimated distance below certain thresholds. 
For the blue goal, the robustness of the algorithm noticeably improved when the value of $M W$ increased. For the goal, the best results were obtained again for a $M W$ value of 0.25 . The behaviour of the algorithm varies for the different objects to be detected when $M W$ increases.

The ball is always captured as a small round orange object and very few frames show the ball partially hidden behind other objects. Because of this, the filtering process gives us useful information for the initialization of the new individuals. The $\langle x, y\rangle$ position of the ball inside a frame will be close to the centroid $<x, y\rangle$ obtained for the orange pixels after the filtering process. If we excessively increase the number of individuals cloned from previous iterations, the number of individuals initialized with the filtering information will be lower than the number needed for optimal convergence.

In spite of these drawbacks, a small percentage of individuals from previous iterations improves the system's convergence, because the algorithm will have a more diverse initial population. The offspring obtained by crossing individuals initialized in different ways will be able to obtain better fitness values. The individuals from previous iterations will be very useful if the initial information (obtained via the filtering process) was noisy.

The situation is completely different for goal detection. The shape of the goals in the frame depends on the position and orientation between camera and goal. The size of a goal's projection is bigger than that obtained for the ball, as can be observed in Fig.6. Individuals that are far from the solution can obtain fitness values greater than zero, due to the useful information stored in their genes. The risk of falling into local optimums is much greater for goal detection and the filtering information is less useful. Initializing individuals in different ways will help the algorithm to escape from local optimums. The solution represented by individuals from previous iterations will usually be closer to the global optimum than the one represented by the individuals initialized with the filtering information, especially for minor changes between frames.

\section{Conclusions and Future Work}

According to the results obtained from the first experiment, our system is a robust alternative to traditional systems for object recognition. It uses the principles of genetic algorithms with a short execution time, which allows the system to operate in real time.

The $\beta$ parameter should always be $\equiv$ ned from the other parameters. This parameter can be correctly obtained ifthe robot's angles are measured without error. The number of genes for the individuals should be as small as possible. Based on the results obtained in the third experiment, the similarity between consecutive frames can be used to improve the performance of our system.

The system was originally developed for goals and ball recognition, but in view of the results obtained and the available alternatives, the main application for the system should be that of goal detection. This is because goal recognition is much more difficult than ball detection, which can be done by using other techniques.

For future work, we aim to integrate the system developed with a localization 
method, such as Montecarlo[11] or Kalman Filters[12]. The selected localization method should use the estimated distances and orientations to the goals and the fitness of the best individual, and in order to integrate the visual and the odometry information in an optimal way[13], the fitness of the best individual could be used to represent the goodness of the visual information.

Adding some restrictions to the initialization of the new individuals by taking into account the robot's estimated pose could also be considered.

\section{References}

1. Wasik, Z., Saffiotti, A.: Robust color segmentation for the robocup domain. Pattern Recognition, Proc. of the Int. Conf. on Pattern Recognition (ICPR) 2 (2002) 651654

2. Rofer, T., Brunn, R., Dahm, I., Hebbel, M., Hoffmann, J., Jungel, M., Laue, T., Lotzsch, M., Nistico, W., Spranger, M.: GermanTeam 2004. Team Report RoboCup (2004)

3. Jüngel, M., Hoffmann, J., Lötzsch, M.: A real-time auto-adjusting vision system for robotic soccer. In: In 7th International Workshop on RoboCup 2003 (Robot World Cup Soccer Games and Conferences), Lecture Notes in Artificial Intelligence, Springer (2004) 214-225

4. Mitchell, M.: An Introduction to Genetic Algorithms. (1996)

5. Whitley, L.: Cellular Genetic Algorithms. In: Proceedings of the 5th International Conference on Genetic Algorithms table of contents, Morgan Kaufmann Publishers Inc. San Francisco, CA, USA (1993)

6. Gouaillier, D., Hugel, V., Blazevic, P., Kilner, C., Monceaux, J., Lafourcade, P., Marnier, B., Serre, J., Maisonnier, B.: The nao humanoid: a combination of performance and affordability. CoRR abs/0807.3223 (2008)

7. Foley, J.D., van Dam, A., Feiner, S.K., Hughes, J.F.: Computer graphics: principles and practice. Addison-Wesley Longman Publishing Co., Inc. (1990)

8. Borenstein, J., Everestt, H., Feng, L.: Where am I? Sensors and Methods for Mobile Robot Positioning. (1996)

9. Bach, J., Jungel, M.: Using pattern matching on a flexible, horizon-aligned grid for robotic vision. Concurrency, Specification and Programming-CSP 1(2002) (2002) $11-19$

10. Moscato, P.: Memetic algorithms: a short introduction. Mcgraw-Hill'S Advanced Topics In Computer Science Series (1999) 219-234

11. Fox, D., Burgard, W., Thrun, S.: Active markov localization for mobile robots (1998)

12. Negenborn, R.: Robot localization and kalman filters. (2003)

13. Jesús Martínez-Gómez, José A. Gámez, I.G.V.: An improved markov-based localization approach by using image quality evaluation. In: Proceedings of the 10th International Conference on Control, Automation, Robotics and Vision (ICARCV). (2008) $1236-1241$ 